\title{
Parasitos intestinais em galinhas caipiras da região metropolitana de Porto Alegre, RS
}

\author{
Gabriela Bernardino de Siqueira1 \& Sandra Márcia Tietz Marques²* \\ ${ }^{1}$ Graduanda de Medicina Veterinária - Faculdade de Veterinária, Universidade Federal do Rio Grande do Sul. \\ Porto Alegre, Rio Grande do Sul, Brasil \\ ${ }^{2}$ Médica Veterinária, Doutora - Faculdade de Veterinária, Departamento de Patologia Clínica Veterinária, \\ Universidade Federal do Rio Grande do Sul, Porto Alegre, Rio Grande do Sul, Brasil \\ *Autor para correspondência, E-mail: sandra.marques@ufrgs.br
}

\begin{abstract}
RESUMO. O objetivo deste estudo foi determinar a prevalência de parasitos intestinais em galinhas caipiras criadas em regime extensivo na região metropolitana de Porto Alegre, RS. Foram analisadas 112 amostras fecais de aves de 14 propriedades e processadas pelas técnicas de flutuação com solução saturada de cloreto de sódio e de sedimentação espontânea. Treze propriedades $(92,9 \%)$ apresentaram aves parasitadas. A prevalência geral foi de 55,4\% (62/112) das aves parasitadas, com ovos de helmintos dos gêneros Capillaria, Heterakis, Ascaridia, Strongyloides, Strongyloidea e protozoários do gênero Eimeria. O único cestóide encontrado foi o do gênero Choanotaenia. Capillaria spp. foi o helminto mais prevalente $(78,6 \%)$ independente de município. O diagnóstico parasitológico pelas amostras fecais é uma ferramenta adequada para auxiliar no controle de verminoses. Embora a prevalência detectada foi alta, os criadores não evidenciaram nenhum problema sanitário nas aves.
\end{abstract}

Palavras chave: agricultura familiar, galinha caipira, parasitos intestinais

\section{Intestinal parasites in free-range chickens in the metropolitan area of Porto Alegre, RS}

\begin{abstract}
The aim of this study was to determine the prevalence of intestinal parasites in free range chicken reared in extensive conditions in the metropolitan region of Porto Alegre. 112 fecal specimens were analyzed poultry properties 14 and processed by flotation techniques with saturated solution of sodium chloride and spontaneous sedimentation. Thirteen properties $(92.9 \%)$ were parasitized birds. The overall prevalence was $55.4 \%(62 / 112)$ of the infected birds with helminth eggs of genus Capillaria, Heterakis, Ascaridia, Strongyloides, Strongyloidea and protozoa of the genus Eimeria. The only cestode found was the genus Choanotaenia. Capillaria sp. was the most prevalent helminth $(78.6 \%)$ independent municipality. The parasitological diagnosis using fecal samples is suitable tool to assist in parasitic control. Although the prevalence detected was high, the creators showed no health problem in poultry.
\end{abstract}

Keywords: free-range chickens, family farming, intestinal parasites

\section{Introdução}

A produção de galinhas caipiras ou galinhas de fundo de quintal no Brasil está presente em cerca de $80 \%$ das propriedades rurais, integrando os recursos de subsistência de grande parte dos pequenos produtores (Sobral et al., 2010). Os requisitos para produção primária das galinhas caipiras requerem acesso em áreas externas para alimentação, exercícios e manifestação de

comportamentos inerentes à espécie. A alimentação deve ser com ração constituída por ingredientes preferencialmente de origem vegetal, sendo proibido o uso de promotores de base antimicrobiana. Apenas linhagens de aves de crescimento lento devem receber esta denominação, sendo vetada a utilização de todos e quaisquer insumos, produtos e medicamentos 
veterinários não autorizados ou não registrados para uso em aves conforme a legislação vigente (NBR 16389) (Azevedo et al., 2016).

Estas criações domésticas, praticadas em unidades agrícolas familiares, se caracterizam pela forma de exploração extensiva na qual inexistem instalações e práticas de manejo que contemplem eficientemente os aspectos reprodutivo, nutricional e sanitário (Quadros et al., 2015); entretanto a avicultura caipira deve atender as condições de criação determinadas pelo Ofício Circular da Divisão de Operações Industriais/Departamento de Inspeção de Produtos de Origem Animal DOI/DIPOA) número 007/99 de 19/05/1999 (MAPA, 2002, 2012).

Nestes sistemas, a ocorrência de infecções parasitárias em aves apresenta prevalência de praticamente $100 \%$ do plantel causando perdas econômicas, como aumento na taxa de mortalidade e nos custos de profilaxia. O diagnóstico parasitológico em galinhas caipiras criadas no Brasil carece de dados atualizados nas diversas regiões brasileiras, principalmente pela extensão territorial, diversidade climática e cultural, bem como pela característica de produção caseira ou de fundo de quintal (Sobral et al., 2010).

Devido aos poucos estudos realizados, o objetivo deste trabalho foi conhecer a prevalência de parasitos intestinais em galinhas caipiras criadas em regime extensivo na região metropolitana de Porto Alegre, RS.

\section{Material e Métodos}

As amostras fecais das aves criadas em regime extensivo avaliadas neste experimento foram originárias de material recebido no Laboratório de Helmintologia pelos criadores no período de cinco meses (dezembro/2015 abril/2016). Foi solicitada a permissão para que os resultados dos exames pudessem ser divulgados. Não se aplica grau de severidade quanto ao bem estar animal ou qualquer conflito ético pois as amostras fecais foram obtidas do solo, poleiros ou instalações. Foram avaliadas 112 amostras fecais de galinhas caipiras de 14 propriedades localizadas nas cidades de Canoas, Novo Hamburgo, Viamão e Porto Alegre, pertencentes a região metropolitana de Porto Alegre, no Estado do Rio Grande do Sul.

Os proprietários realizaram as coletas das fezes, acondicionaram em embalagens plásticas, preservaram na geladeira e levaram no laboratório no prazo máximo de 48 horas.

No recebimento das amostras, há uma ficha de acompanhamento com preenchimento da resenha pelos clientes. Em todas as propriedades, as aves permanecem em pátio de terra e gramado. Algumas das propriedades deixam acesso livre das aves a outros animais, como cães ou gatos. Quando anoitece, as aves repousam em instalações abertas porém com cobertura de telhado. Os pátios são limpos com regularidade e as fezes recolhidas são utilizadas na adubação de hortas e plantações. As propriedades possuem de 400 até $3000 \mathrm{~m}^{2}$ de área e o número médio de aves é de 15,4. As aves são alimentadas com ração para aves de postura, milho, sobras de hortaliças e frutas e não são submetidas a tratamento anti-helmíntico convencional. As raças criadas são New Hampshire e Rhodes Island cujo foco da produção é a obtenção de ovos, o excedente é vendido e as galinhas poedeiras de descarte são abatidas para $\mathrm{o}$ consumo.

As fezes foram processadas pelas das técnicas de sedimentação espontânea (Hoffmann, Pons e Janer) e flutuação com solução saturada de cloreto de sódio (Willis-Mollay) descritos por Hendrix \& Robinson (2014). As amostras foram visualizadas em microscopia óptica (Microscópio Nikon, Japan), sob objetivas 10x e 40x.

\section{Resultados e discussão}

Das pequenas criações caseiras que solicitaram avaliação parasitológica, 92,9\% (13/14) apresentaram aves parasitadas, com $84,6 \%$ para a infecção por Capillaria sp. A prevalência geral foi de 55,4\% (62/112). O cestóide do gênero Choanotaenia foi identificado através da técnica de sedimentação espontânea e os demais gêneros foram identificados preferencialmente pela técnica de flutuação. A Tabela 1 apresenta os resultados das frequências positivas para os diversos municípios da região metropolitana. A Tabela 2 mostra a frequência de positividade para helmintos e protozoários.

Os sinais clínicos sugestivos de parasitoses intestinais evidenciam fraqueza, anemia, perda de apetite, diarreia e emagrecimento. A detecção de oocistos ou ovos de parasitos em amostras fecais não necessariamente reflete a existência de lesões na mucosa do trato digestivo, e depende de fatores como a carga parasitária, o tipo de parasito, a idade do hospedeiro, bem como seu 
estado imunológico (Gomes et al., 2009). Os cestóides são facilmente encontrados no tipo de criação extensiva, pois requerem a presença de hospedeiros intermediários (moluscos gastrópodes, moscas, minhocas, formigas e outros insetos coprófagos) enquanto Capillaria sp., Eimeria sp., Ascaridia galli e Heterakis gallinarum tem ciclo direto. $H$. gallinarum tem importância por veicular um protozoário denominado Histomonas meleagridis, agente da chamada histomonose ou "cabeça preta" das galinhas (Cardozo and Yamamura, 2004).

Tabela 1. Distribuição de propriedades por município (área urbana e rural) e prevalência de parasitismo intestinal em galinhas caipiras da região metropolitana de Porto Alegre, Rio Grande do Sul, no período de dezembro de 2015 a abril de 2016.

\begin{tabular}{lccccc}
\hline & \multicolumn{3}{c}{ Área } & \multicolumn{3}{c}{ Amostras avaliadas } \\
\cline { 2 - 6 } Local & Urbana & Rural & Total & Positivas & $\%$ \\
\hline Viamão & 1 & 4 & 32 & 24 & 75 \\
Porto Alegre & 4 & 1 & 31 & 27 & 87 \\
Canoas & 3 & - & 25 & 2 & 8 \\
Novo Hamburgo & 1 & - & 24 & 9 & 37,5 \\
TOTAL & 9 & 5 & 112 & 62 & 55,4 \\
\hline
\end{tabular}

Tabela 2. Frequência de ovos e oocistos de parasitos diagnosticados por duas técnicas coprológicas em galinhas caipiras de quatro municípios da região metropolitana de Porto Alegre, RS, de dezembro de 2015 a abril de 2016.

\begin{tabular}{lllll}
\hline Propriedade & Amostras & Positivas & $\%$ & Ovos/oocistos de parasitos \\
\hline 1 & 5 & 1 & 20 & Strongyloidea \\
2 & 3 & 2 & 67 & Capillaria sp. \\
3 & 10 & 10 & 100 & Capillaria sp.; Eimeria sp.; Strongyloidea \\
4 & 6 & 5 & 83 & Capillaria sp.; Eimeria sp.; Heterakis gallinarum \\
5 & 5 & 5 & 100 & Capillaria sp.; Eimeria spp.; Choanotaenia sp. \\
6 & 6 & 4 & 67 & Capillaria sp. \\
7 & 10 & 7 & 70 & Capillaria sp.; Eimeria sp.; Heterakis gallinarum \\
8 & 6 & 6 & 100 & Capillaria spp.; Eimeria sp.; Heterakis gallinarum; Strongyloidea \\
9 & 9 & 1 & 11 & Ascaridia galli \\
10 & 5 & 0 & 0 & - \\
11 & 4 & 4 & 100 & Capillaria sp.; Eimeria sp.; Choanotaenia sp. \\
12 & 11 & 1 & 9 & Capillaria sp. \\
13 & 24 & 9 & 37 & Capillaria sp. \\
14 & 8 & 7 & 87 & Capillaria sp.; Heterakis gallinarum; Strongyloides sp. \\
\hline
\end{tabular}

Os resultados desta pesquisa mostram a presença de helmintos do gênero Capillaria, Heterakis, Choanotaenia, Strongyloides, Strongyloidea e protozoário do gênero Eimeria em galinhas caipiras criadas no sistema extensivo em propriedades na cidade de Porto Alegre, cujas frequências variaram de $80 \%$ a $100 \%$ com predomínio de aves multi-infectadas. Para as amostras fecais de galinhas criadas no município de Viamão a presença de parasitos variou de $11 \%$ até $100 \%$, com predomínio de ovos de Capillaria sp. e oocistos de Eimeria sp. As excretas das aves 
de Canoas e Novo Hamburgo submetidas a duas técnicas parasitológicas apresentaram somente ovos de Capillaria sp.

Comparando estes dados com estudo de Albuquerque (1997) em galinhas de fundo de quintal em Porto Alegre (RS), após quase 40 anos de intervalo, Capillaria sp. também foi o helminto mais diagnosticado (86\%) seguido por H. gallinarum $(81 \%)$.

As amostras analisadas mostraram uma prevalência de $55,4 \%$, superior ao resultado obtido por Quadros et al. (2015) em aves da região serrana de Santa Catarina, onde as condições de manejo e de clima se assemelham, entretanto Brandão et al. (2008), no semi-árido paraibano e Gomes et al. (2009) no Rio de Janeiro demonstraram, respectivamente, prevalências de 68\% e 67\%. Vieira (2010) analisando vísceras de galinhas em sistema colonial caipira, no estado do Paraná, detectou, de um total de 262 frangos para consumo, os helmintos $H$. gallinarum, A. galli e Capillaria sp. como os mais prevalentes. Vita et al. (2014) avaliando infecção natural em aves caipiras criadas no Rio de Janeiro identificaram os gêneros de helmintos Ascaridia (35\%), Capillaria (30\%), Heterakis (25\%) e Strongyloides (10\%). Silva et al. (2016) investigando parasitos em galinhas de 17 municípios de São Paulo e diagnosticaram Ascaridia galli (71\%), Capillaria sp. (59\%), $H$. gallinarum (76\%), Strongyloides sp. (6\%) e Choanotaenia infundibulum (88\%), valores de frequência similares com estudos ao redor do mundo.

Na comparação com resultados obtidos de galinhas de criação extensiva fora do Brasil, $\underline{\text { Kurt }}$ \& Acici (2008) identificaram maior ocorrência dos gêneros Heterakis e Capillaria na Turquia. Resultados semelhantes também foram confirmados em análises parasitológicas na Tanzânia (Magwisha et al., 2002) e Etiópia (Eshetu et al., 2001). Prevalências superiores a encontradas neste trabalho foram evidenciadas no Iran $(100 \%)$ por Naem \& Eskandari (2005); em Marrocos $(89,9 \%)$ por Hassouni \& Belghyti (2006); na Nigéria $(87,8 \%)$ por Yoriyo et al. (2008); também na Nigéria, Nnadi \& George (2010) registraram prevalência de $35,5 \%$ para helmintos, sendo Ascaridia galli e H. gallinarum os mais frequentes (35-40\%), além de Eimeria sp. (16\%); e na Índia (100\%) por Roy (2009). Na África do Sul o nematódeo mais prevalente diagnosticado por Mukaratirwa \& Khumalo (2010) foi $H$. gallinarum $(80-94,4 \%)$.

São poucos os estudos publicados sobre o perfil parasitológico em galinhas caipiras, o que torna relevante este estudo mostrando uma fauna parasitária semelhante do último relato para Porto Alegre, contando com a capilariose como a infecção mais prevalente. Os criadores destas aves em quintal relataram que não utilizam nenhum vermífugo e tem a percepção de que as galinhas são saudáveis, além de casos de óbito serem raros. Embora parasitadas, as aves apresentam resistência, provavelmente pelo fato de estarem soltas, isentas de stress ou apresentarem um grau de infecção baixo.

Em relação a eimeriose os criadores não notaram problemas sanitários, o que corrobora relatos de literatura, onde a coccidiose tende a ser mais patogênica em produções de confinamento de ciclo curto. Kaboudi et al. (2016) avaliaram por necropsia, as alterações decorrentes da coccidiose clínica e subclínica em galinhas criadas soltas na Tunísia. A média na prevalência das lesões (12\%), somente diferiu entre as estações do ano, com $3,1 \%$ no verão e $50,9 \%$ no outono, embora os índices foram semelhantes para o grupo sintomático e subclínico. Este trabalho identificou no verão a presença de oocistos de Eimeria sp. na forma subclínica $(42,8 \%)$. Os criadores destas aves relataram que, seguindo a sabedoria popular, é comum utilizarem sementes frutíferas e plantas para prevenir e tratar as parasitoses, destacando-se as sementes de abóbora, mamão, melancia e melão, bem como casca de banana, de laranja e limão, capim cidreira, hortelã e tomilho. Barbosa et al. (2008) recomendaram como alternativa de vermífugos naturais a utilização de sementes de melancia, mamão, melão e perfilhos de bananeira a vontade.

Métodos de exames laboratoriais baseados na pesquisa de ovos e oocistos nas fezes de aves domésticas devem ser incentivados e realizados a fim de contribuir para o estabelecimento de controle estratégico em criações extensivas. As técnicas parasitológicas utilizadas na rotina laboratorial são simples, pouco onerosas e de fácil execução, sendo também uma boa alternativa à necropsia nos casos em que não há óbitos (Gomes et al., 2009). 


\section{Conclusão}

Capillaria sp. foi o helminto mais prevalente independente de município, seguido por Eimeria sp. Os gêneros detectados foram Capillaria, Heterakis, Choanotaenia, Ascaridia, Stronyloidea, Strongyloides e o protozoário Eimeria, entretanto as aves não apresentavam sinais clínicos sugestivos de infecção parasitária.

Agradecimento: Ao Prof. Dr. André Carissimi pelas sugestões e revisão do artigo.

\section{Referências Bibliográficas}

Albuquerque, B. D. L. (1997). Fauna helmíntica de Gallus gallus domesticus (Lin.) de criação em fundo de quintal em Porto Alegre-RS. 1977, Universidade Federal do Rio Grande do Sul, Porto Alegre.

Azevedo, G. S., Souza, J. P. L, Cardoso, J. A., Araujo, P. H. H., Santos Neta, E. R. \& Novas, M. P. V. (2016). Produção de aves em sistema orgânico. Pubvet: Publicações em Medicina Veterinária e Zootecnia, 10, 327333.

Barbosa, F. J. V., Nascimento, M., Diniz, F., Nascimento Hoston, T. S. \& Araújo Neto, R. B. (2008). Sistema alternativo de criação de galinhas caipiras Embrapa Meio-NorteSistema de Produção (INFOTECA-E). p 1-69, Teresina - Piauí.

Brandão, P., Sobral, E. S., Brito, I. C. A., Silva, S. G., Silva, I. K. C. \& Costa V. M. M. (2008). Prevalência de endoparasitoses em galinha caipira em assentamento rural no semi-árido paraibano. In: Anais 5o Congresso Nordestino de Produção Animal, Aracaju, SE.

Cardozo, S. P. \& Yamamura, M. H. (2004). Parasitas em produção de frangos no sistema de criação tipo colonial/caipira no Brasil. Semina: Ciências Agrarias, 25, 63-74.

Eshetu, Y., Mulualem, E., Ibrahim, H., Berhanu, A. \& Aberra, K. (2001). Study of gastrointestinal helminths of scavenging chickens in four rural districts of Amhara region, Ethiopia. Revue Scientifique et Technique, 20, 791-796.

Gomes, F. F., Machado, H., Lemos, L., Almeida, L. \& Daher, R. (2009). Principais parasitos intestinais diagnosticados em galinhas domésticas criadas em regime extensivo na municipalidade de Campos dos Goytacazes, RJ. Ciência Animal Brasileira, 10, 818-822.

Hassouni, T. \& Belghyti, D. (2006). Distribution of gastrointestinal helminths in chicken farms in the Gharb region-Morocco. Parasitology Research, 99, 181-183.

Hendrix, C. M. \& Robinson, E. D. (2014). Diagnostic parasitology for veterinary technicians. Elsevier Health Sciences.

Kaboudi, K., Umar, S. \& Munir, M. T. (2016). Prevalence of Coccidiosis in Free-Range Chicken in Sidi Thabet, Tunisia. Scientifica 2016, 1-6.

Kurt, M. \& Acici, M. (2008). Cross-sectional survey on helminth infections of chickens in the Samsun region, Turkey. Deutsche tIerarztliche Wochenschrift 115, 239-242.

Magwisha, H., Kassuku, A., Kyvsgaard, N. \& Permin, A. (2002). A comparison of the prevalence and burdens of helminth infections in growers and adult free-range chickens. Tropical Animal Health and Production, 34, 205-214.

MAPA. Ministério da Agricultura, Pecuária e Abastecimento, Brasil. (2002). Departamento de Inspeção de Produtos de Origem Animal/ divisão de operações industriais. Ofício Circular DOI/DIPOA $n^{\circ} 007 / 99 d e$ 19/05/1999. Registro do Produto "Frango Caipira ou Frango Colonial" ou "Frango Tipo ou Estilo Caipira" ou "Tipo ou Estilo Colonial". Brasília, DF.

MAPA. Ministério da Agricultura, Pecuária e Abastecimento, Brasil. (2012). Departamento de Inspeção de Produtos de Origem Animal/ divisão de operações industriais. Ofício Circular DOI/DIPOA $n^{\circ}$ 02/2012 de 01/02/2012. Registro do Produto "Frango Caipira ou Frango Colonial" ou "Frango Tipo ou Estilo Caipira" ou "Tipo ou Estilo Colonial". Brasília, DF.

Mukaratirwa, S. \& Khumalo, M. P. (2010). Prevalence of helminth parasites in free-range chickens from selected rural communities in KwaZulu-Natal province of South Africa. Journal of the South African Veterinary Association, 81, 97-101.

Naem, S. \& Eskandari, S. (2005). Prevalence of intestinal helminths of native chickens in Urmia, Iran. Iranian Journal Veterinary Research University Shiraz, 3, 200-203. 
Nnadi, P. A. \& George, S. O. (20100. A crosssectional survey on parasites of chickens in selected villages in the subhumid zones of South-Eastern Nigeria. Journal of Parasitology Research, 2010, 1-6.

Quadros, R. M., Wiggers, S. B., Paes, M. P. V. \& Marques, S. M. T. (2015). Prevalence of endoparasites and ectoparasites in free-range hens in small holdings in the state of Santa Catarina, Brazil. Pubvet: Publicações em Medicina Veterinaria e Zootecnia, 9, 1-5.

Roy, D. K. (2009). Helminthosis of free-range chickens in Bangladesh-with emphasis on prevalence end effect on productivity, The Royal Veterinary and Agricultural University, 38p.

Silva, G. S., Romera, D. M., Fonseca, L. E. C. \& Meireles, M. V. (2016). Helminthic parasites of chickens (Gallus domesticus) in different regions of São Paulo State, Brazil. Revista Brasileira de Ciência Avícola, 18, 163-168.

Sobral, F. E. S., Brandão, P. A. \& Athayde, A. C. R. (2010). Utilização de fitoterápicos no tratamento de parasitoses em galinhas caipira criadas em sistema semi-extensivo. Agropecuária Científica do Semi-Árido, 6, 16.

Vieira, F. E. G. (2010). Helmintofauna em frangos (Gallus gallus domesticus
LINNAEUS, 1758) criados em sistema colonial/caipira na região norte do Estado do Paraná [mestrado]. 2010 Universidade Estadual de Londrina, Londrina.

Vita, G. F., Ferreira, I., Costa Pereira, M. A. V., Azevedo, J. R., Sanavria, A., Barbosa, C. G., Gallo, S. S. M. \& Vasconcellos, H. V. G. (2014). Eficácia de Chenopodium ambrosioides (erva-de-santa-maria) no controle de endoparasitos de Gallus gallus (galinha caipira). Pesquisa Veterinária Brasileira, 34, 39-45.

Yoriyo, K. P., Adang, K. L., Fabiyi, J. P. \& Adamu, S. U. (2008). Helminthes parasites of local chickens in Bauchi State, Nigeria. Science World Journal, 3, 35-37.

\section{Article History}

Received 22 June 2016

Accepted 12 July 2016

Available on line 27 July 2016

License information: This is an open-access article distributed under the terms of the Creative Commons Attribution License, which permits unrestricted use, distribution, and reproduction in any medium, provided the original work is properly cited. 